\title{
Les nouveaux enjeux de l'éducation musicale au collège
}

Denis Waleckx

\section{OpenEdition}

1 Journals

Édition électronique

URL : http://journals.openedition.org/trema/362

DOI : $10.4000 /$ trema.362

ISSN : 2107-0997

Éditeur

Faculté d'Éducation de l'université de Montpellier

\section{Édition imprimée}

Date de publication : 1 décembre 2005

Pagination : 43-48

ISSN : 1167-315X

\section{Référence électronique}

Denis Waleckx, «Les nouveaux enjeux de l'éducation musicale au collège », Tréma [En ligne], 25 | 2005, mis en ligne le 06 janvier 2010, consulté le 30 avril 2019. URL : http://journals.openedition.org/ trema/362 ; DOI : 10.4000/trema.362

Ce document a été généré automatiquement le 30 avril 2019.

Trema 


\title{
Les nouveaux enjeux de l'éducation musicale au collège
}

\author{
Denis Waleckx
}

L'éducation musicale n'est certainement pas la seule discipline à avoir connu une profonde mutation durant les dernières décennies, devant répondre au défi de la massification de l'enseignement et aux nécessités du collège unique, tout en préservant les fondamentaux liés à sa spécificité. Mais l'évolution qui la touche, si elle n'est pas récente, a connu depuis quelques années une accélération. Celle-ci, même si elle a été pilotée et fortement accompagnée, s'est unanimement imposée et donne à la discipline une meilleure lisibilité, ce qui n'empêche malheureusement pas les représentations réductrices, parfois caricaturales, restant trop fréquemment accolées à l'enseignement de l'éducation musicale, mais rend ces représentations encore moins fondées et donc plus insignifiantes. Alors que Claude THELOT a remis son rapport sur l'école ${ }^{1}$, alors qu'une nouvelle loi d'orientation entrera en application à la rentrée $2006^{2}$, alors que chacune des disciplines est interrogée au regard de sa participation au socle commun de connaissances $^{3}$, il nous parait opportun de rappeler ce que sont ces enjeux. Après un rappel rapide de qui fait la réalité des évolutions et les lignes de force de l'éducation musicale au collège, nous mettrons en exergue son apport spécifique et sa contribution à la formation générale de l'élève. Nous conclurons en explicitant ce que ces enjeux impliquent en terme de positionnement éducatif pour le professeur d'aujourd'hui.

\section{Une discipline obligatoire ; des professeurs spécialistes plus jeunes que la moyenne}

2 Au collège, l'éducation musicale est une discipline obligatoire comme elle l'est d'ailleurs à l'école. Mais, alors que dans le premier degré, elle est portée par un instituteur ou un professeur des écoles polyvalent éventuellement assisté d'intervenants extérieurs, son enseignement est confié, dans le second degré, à des professeurs spécialistes (certifiés d'éducation musicale et de chant choral ou agrégés de musique). 
3 Les professeurs d'éducation musicale sont en moyenne plus jeune d'environ quatre ans que leurs collègues des autres disciplines ${ }^{4}$ et si l'éducation musicale est touchée par la féminisation, elle l'est plutôt moins que d'autres matières (58 \% en 2003-2004 contre 63,3 toutes disciplines confondues dans le second degré) $)^{5}$. Ces deux caractéristiques fortes du corps de professeurs d'éducation musicale - équilibre entre les deux sexes et relative jeunesse - sont deux atouts importants pour les évolutions à venir.

\section{Une évolution tangible touchant l'ensemble des programmes...}

4 L'ensemble des programmes d'enseignement du second degré a été revu entre 1996 (date d'entrée en vigueur du nouveau programme de 6ème ) et 2001 (nouveau programme de terminale), touchant ainsi tous les niveaux du second degré. Loin de désorganiser ou de fragiliser son enseignement, ces modifications successives, s'appuyant sur l'expérience acquise et mutualisant les réflexions menées auparavant, donnent de la discipline une image très cohérente, où s'imposent des éléments saillants.

\section{III. ...et générant une meilleure lisibilité}

5 Les objectifs d'enseignement de la discipline sont ainsi clairement réaffirmés : il s'agit de développer la sensibilité esthétique de l'élève, d'établir des repères culturels et émotionnels, d'affiner sa capacité d'expression et d'invention. Percevoir, connaître, produire sont donc trois postures fondamentales de l'élève en cours d'éducation musicale. D'autres traits se dessinent clairement :

6 L'éducation musicale est fondée sur des pratiques : chanter, écouter, jouer, créer. Parmi celles-ci, les deux premières s'imposent comme essentielles, relevant d'objectifs de formation, les deux dernières restant davantage des outils au service des objectifs poursuivis.

7 - Ces pratiques naissent d'une confrontation avec des œuvres d'esthétique et de genre diversifiés. L'élargissement du répertoire à des œuvres jusqu'alors absentes ou peu présentes est un des éléments majeurs de l'évolution des pratiques pédagogiques (usage qui tend à se banaliser d'œuvres contemporaines, voire très contemporaines, de jazz, de musiques traditionnelles, de musiques savantes ou populaires...). A ce titre, si les programmes invitent aujourd'hui à la confrontation d'œuvres d'esthétique et de genre différents, c'est pour encourager la mise en relief de rapprochements possibles ou de constantes dans l'organisation du langage de la musique.

8 - Si la discipline vise à la transmission d'un savoir spécifique, elle a recentré sa réflexion et sa communication sur les compétences qu'elle permet de développer chez les élèves. Certaines de ces compétences sont musicales (utiliser et maîtriser sa voix parlée et chantée, interpréter, tenir sa place dans une réalisation collective, inventer, exprimer sa sensibilité, percevoir et rendre compte des composantes musicales à l'aide d'un vocabulaire approprié, etc.), d'autres sont transversales (mémoriser, traiter de l'information, maîtriser des langages, se situer dans un groupe et y participer activement, etc.). 
9 - La recherche de cohérence entre les activités est fortement préconisée. Elle permet d'éviter un fractionnement supplémentaire du temps scolaire, de mieux articuler les activités entre elles, et d'ouvrir éventuellement à d'autres champs de la connaissance. L'organisation de l'enseignement autour de thématiques centrales porteuses de sens, imposées au lycée, possibles au collège, illustre cette recherche. A cette cohésion horizontale répond une cohérence verticale entre le collège et le lycée. Les enseignements au lycée concernent tous les élèves issus du collège qui, quel que soit leur profil, souhaitent poursuivre une formation en éducation musicale. Cette exigence impérieuse impose une continuité entre les deux niveaux d'enseignement. Un autre levier vient de l'usage raisonnable et raisonné en collège du programme limitatif du baccalauréat qui présente le triple avantage de favoriser l'élaboration d'une culture commune à tous les professeurs d'éducation musicale quel que soit leur niveau d'enseignement, d'encourager un usage généralisé des démarches pédagogiques et outils que ce programme génère, et de garantir une meilleure évaluation au baccalauréat en participant de la formation des membres du jury.

\section{Des maquettes de concours de recrutement réactualisées}

10 Il fallait bien que l'institution se donne les moyens de recruter les personnels capables d'accompagner pertinemment les mutations de la discipline. Les maquettes aux épreuves des concours ont donc logiquement évolué, tournées vers une professionnalité qui se devait d'être indiscutable, c'est-à-dire qui réaffirme les spécificités disciplinaires " historiques » mais aussi capable de répondre aux nouveaux défis éducatifs. Les épreuves d'admission du CAPES sont à ce titre révélatrices de cette recherche d'équilibre : on peut y souligner tout à la fois l'importance de l'évaluation des compétences vocales des candidats et la présence d'une épreuve de direction de chœur (pour un CAPES d'éducation musicale et de chant choral) mais aussi celle d'une épreuve d'arrangement, pour laquelle l'usage de l'informatique, pourtant non obligatoire, se retrouve de fait plébiscité par les candidats, grâce à la bonne connaissance de ses grandes potentialités.

\section{L'éducation musicale est actuellement interrogée dans sa spécificité...}

11 Cette interrogation, qui touche, rappelons-le, l'ensemble des disciplines est actuellement réactualisée par la nécessité de définition d'un socle commun de connaissances. L'éducation musicale est, avec les arts plastiques, un des deux piliers de l'enseignement artistique, réaffirmés récemment comme tels : dans le cadre de la classe de troisième, elle fait effectivement partie des enseignements obligatoires pour tous ${ }^{6}$. Au-delà de cette importante reconnaissance institutionnelle, la discipline possède bien un objet d'étude spécifique et exclusif qui lui confère une légitimité indiscutable à participer au socle commun nécessaire à tous les élèves: il s'agit de l'exploration du monde sonore et du travail sur des organes essentiels de la communication que sont les appareils phonatoire et auditif, le domaine de la perception sonore étant, avec le domaine visuel, au centre des relations humaines. 


\section{VI. ...et dans sa capacité particulière à participer aux fondamentaux éducatifs transversaux}

12 L'éducation musicale est aussi un vecteur performant de transversalité : si l'exploration $\mathrm{du}$ monde sonore génère un type particulier de «traitement de l'information", l'éducation musicale contribue de manière essentielle à la maîtrise des langages. Un long passage des documents d'accompagnement des programmes du cycle central est d'ailleurs consacré à la contribution de l'éducation musicale à la maîtrise de la langue française ${ }^{7}$, rappelant l'enrichissement du vocabulaire et de la syntaxe que peut engendrer l'apprentissage bien conduit d'un chant ou l'élargissement du bagage linguistique qui s'opère progressivement lors de l'activité d'écoute, lorsqu'il s'agit d'apprendre à exprimer ses émotions ou à rendre compte des phénomènes entendus.

13 L'éducation musicale participe également largement à l'apprentissage de la citoyenneté en permettant à l'élève de s'impliquer dans des réalisations collectives, en l'amenant à écouter et accepter l'autre, et à s'ouvrir à la diversité des cultures. S'il est vrai qu'un professeur d'éducation musicale n'enseigne aux élèves d'une même classe qu'une heure par semaine, son service de quinze ou de dix-huit heures le met en présence d'un nombre conséquent d'élèves, en général entre quatre cents et cinq cent cinquante élèves. L'influence qu'il exerce sur ses classes a un impact important sur l'ensemble de son établissement. D'autant que la connaissance « large » que l'enseignant a de ses élèves va s'inscrire dans la durée, et qu'en conséquence, le professeur peut percevoir l'évolution des individus et en rendre compte dans la micro société que constitue le collège. Il est d'ailleurs à noter que les programmes n'ignorent pas cet aspect essentiel de la discipline créatrice de lien social, rappelant en préambule que « La fonction du professeur d'éducation musicale dépasse le cadre strict de sa classe : en prolongement des cours, il anime des activités de pratiques collectives (chorale, ensemble instrumental, atelier) et associe ses efforts à ceux des autres membres de l'équipe éducative afin de faire du collège un véritable lieu de vie artistique ${ }^{8}$. La chorale au collège, enseignement facultatif complémentaire, basée sur une démarche de projet inscrite dans le volet culturel du projet d'établissement, prolonge l'éducation musicale obligatoire en approfondissant et en développant certains de ses objectifs. Ouverte sans exigence de pré - requis à tous les jeunes désireux de pratiquer la musique, la chorale est inter niveaux. Elle contribue à l'intégration d'élèves hétérogènes, à leur construction identitaire et au rayonnement des établissements scolaires.

\section{Une implication aisée dans les dispositifs transversaux}

14 Par ailleurs, les itinéraires de découverte (IDD) sont un dispositif nouveau qui a vu le jour au cycle central du collège à la rentrée 2002. Favorisant le travail en équipe des enseignants, sorte d'équivalent au niveau du collège des travaux personnels encadrés (TPE) au lycée, leur finalité est de décloisonner les savoirs, les aptitudes, les talents des élèves et de développer le goût d'apprendre grâce à une approche interdisciplinaire de certaines parties des programmes disciplinaires. Travail autonome des élèves sur des projets, réalisation d'une production individuelle ou collective, initiation à la recherche documentaire et au traitement de l'information en sont les principes essentiels. Quatre 
domaines en définissent le contenu, (la nature et le corps humain, les arts et les humanités, les langues et les civilisations, la création et les techniques). La capacité de l'éducation musicale à s'ouvrir au monde, son aisance à traiter disciplinairement des problématiques ou des thématiques non strictement musicales, le type d'évaluation qui prévaut dans le domaine artistique, s'intéressant à la production finale mais aussi à la démarche en amont qui y a conduit, sont autant d'éléments qui facilitent l'implication de l'éducation musicale dans les itinéraires de découverte.

\section{Une exploration d'autres champs artistiques en troisième?}

15 La logique de la diversification des parcours des élèves s'impose peu à peu, dans un collège qui, selon l'expression d'un ancien ministre, doit à la fois s'adresser “à tous et à chacun“. Pour la classe de troisième, fortement interrogée par cette dialectique, des propositions se dessinent : " Entre l'ambition républicaine de donner à chacun un socle commun de connaissances sinon une culture et des références communes et le besoin de prendre en compte la diversité des talents et des motivations, la nouvelle classe de $3 e$ propose un compromis qui ne vaudra que par la façon dont il sera investi par les équipes éducatives. $»^{9}$

En effet, entre le collège, où les seules disciplines artistiques enseignées sont les arts plastiques et l'éducation musicale, et le lycée qui propose une palette d'enseignements optionnels comprenant sept domaines (arts plastiques, arts appliqués, cinéma audiovisuel, danse, histoire des arts, musique, théâtre et expression dramatique), la classe de troisième, cycle d'orientation, est probablement le moment opportun d'une exploration privilégiée, dans le cadre des enseignements existants, d'autres champs artistiques : "N'y aurait-il alors quelque logique à proposer aux élèves de $3 e$, sortant du même coup du cadre traditionnel des programmes disciplinaires, un choix de projets thématiques inscrits dans la logique des différents domaines enseignés au lycée? L'éducation musicale en 3e ne pourrait-elle devenir un moment de réalisation de projets, mobilisant la formation acquise au bénéfice de l'étude d'une ou plusieurs thématiques inscrites clairement dans les horizons ouverts au lycée ?» ${ }^{10}$

\section{Pour relever les défis, une posture éducative exigeante}

17 S'approprier l'espace classe qui doit rendre propice la pratique musicale, user d'une palette d'outils diversifiés et intégrés à ses pratiques pédagogiques, être porteur d'une cohérence entre toutes les activités du cours, suivre des objectifs d'enseignement clairement identifiés, mettre du liant éducatif au sein de son établissement sont autant de nécessités qui s'imposent aujourd'hui au professeur d'éducation musicale. Rénovée, réaffirmée dans ses fondamentaux disciplinaires et transversaux, la discipline ne manque pas d'atouts. Accompagner les professeurs à toujours mieux connaitre, toujours mieux communiquer, toujours mieux exploiter l'ensemble de ces atouts est un levier fondamental de réussite. 


\section{NOTES}

1. Pour la réussite de tous les élèves, rapport de la Commission du débat national sur l'avenir de l'école, remis par son président Claude THELOT au Premier ministre le 12 octobre 2004, consultable et téléchargeable sur le site http ://www.debatnational.education.fr/

2. http ://www.loi.ecole.gouv.fr/

3. Il s'agit du socle commun de connaissances que chaque élève doit acquérir au cours de la scolarité obligatoire. Sa définition est une des grandes priorités de la loi d'orientation.

4. L'âge moyen des professeurs d'éducation musicale est de 39 ans, celui des professeurs du second degré toutes disciplines confondues était de 42,8 en 2003-2004 (Repères et Références statistiques sur les enseignements, la formation et la recherche 2004, DEP, p. 230).

5. Repères et Références statistiques sur les enseignements, la formation et la recherche 2004, DEP, p. 245.

6. Organisation des enseignements du cycle d'orientation de collège (classe de troisième), arrêté du 2 juillet 2004, JO du 6 juillet 2004, BO n² 28 du 15 juillet 2004.

7. Contribution de l'éducation musicale à la maîtrise de la langue française in Documents d'accompagnement des programmes du cycle central; Programme du cycle central arrêté du 10-1 1997 ; JO du 21-1-1997 ; BO n 5 du 30-1-97.

8. Programme de 6e, arrêté du 22-11-1995, JO nº 278 du 30-11-1995

9. MAESTRACCI, Vincent, IGEN, lettre du 25 octobre 2004. http://www.educnet.education.fr/ musique/infos/lettre.htm, lettre du 25 octobre 2004.

10. Ibid

\section{RÉSUMÉS}

Le collège unique a conduit depuis trente ans l'ensemble des disciplines, et notamment l'éducation musicale, à répondre de manière novatrice au contexte d'un enseignement de masse. Denis Waleckx observe l'apport des directives nationales qui incitent les enseignants à varier les approches et les répertoires. Il insiste aussi sur les nouvelles modalités de recrutement aux concours des enseignants destinées à accompagner les mutations de la discipline.

Within the frame of comprehensive schools ("collège unique") mass-teaching has brought a renewal of teaching methods for all the subjects in the national curriculum. Denis Waleckx examines how national guidelines give the teachers the impulse to vary their approaches and the repertoire. He also underlines the new modes of recruitment at the teachers' competitive examination, which are meant to follow the various changes in music teaching. 


\section{AUTEUR}

\section{DENIS WALECKX}

IA-IPR Montpellier 